\title{
3-Hydroxy-4-\{[(4-methoxyphenyl)imino]methyl $\}$ phenyl Octadecanoate
}

\author{
Sie-Tiong Ha ${ }^{1, *}$, Jun-Kit Beh ${ }^{2}$, Kok-Leei Foo ${ }^{1,2}$, Teck-Ming Koh ${ }^{2, \dagger}$ and Siew-Teng Ong ${ }^{1}$ \\ 1 Department of Chemical Science, Faculty of Science, Universiti Tunku Abdul Rahman, Jln \\ Universiti, Bandar Barat, 31900 Kampar, Perak, Malaysia \\ 2 Department of Science, Faculty of Engineering and Science, Universiti Tunku Abdul Rahman, Jln \\ Genting Kelang, Setapak, 53300 Kuala Lumpur, Malaysia
}

$\dagger$ Present address: Department of Chemistry, National University of Singapore, 3 Science Drive 3, Singapore 117543.

* Author to whom correspondence should be addressed; E-Mail: hast@utar.edu.my or hast_utar@yahoo.com.

Received: 2 April 2010 / Accepted: 4 June 2010 / Published: 8 June 2010

\begin{abstract}
A new Schiff base ester, 3-hydroxy-4-\{[(4-methoxyphenyl)imino]methyl $\}$ phenyl octadecanoate, was synthesized and its IR, ${ }^{1} \mathrm{H}$ NMR, ${ }^{13} \mathrm{C}$ NMR and MS spectroscopic data are presented.
\end{abstract}

Keywords: 3-hydroxy-4-\{[(4-methoxyphenyl)imino]methyl $\}$ phenyl octadecanoate; Schiff base; alkyl chain

Schiff bases have attracted overwhelming attention from many researchers owing to their importance in exhibiting thermochromism and photochromism [1-5]. The presence of a long alkyl chain at the para position of the aldehyde or aniline fragment of $N$-benzylideneanilines has been regarded as one of the important elements which favours the existence of liquid crystal phases [6-8]. Different alkyl chain length can significantly influence the anisotropic properties of liquid crystals [6]. Thus, we report here another new derivative containing an octadecanoyloxy chain, 3-hydroxy-4-\{[(4-methoxyphenyl)imino]methyl $\}$ phenyl octadecanoate. 
Scheme 1. Synthesis of 3-hydroxy-4-\{[(4-methoxyphenyl)imino]methyl $\}$ phenyl octadecanoate.

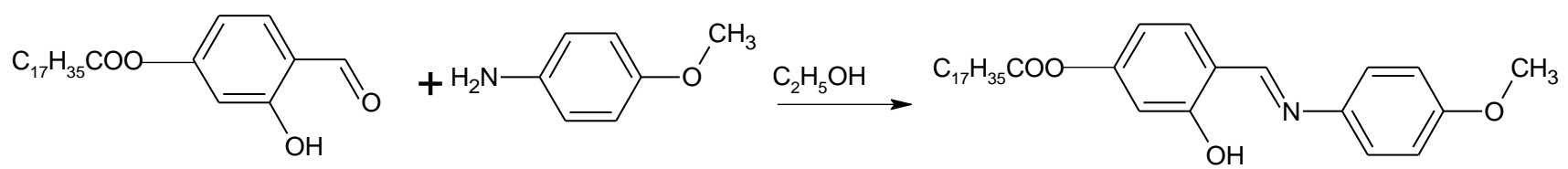

\section{Experimental}

4-Formyl-3-hydroxyphenyl octadecanoate was previously prepared via Steglich esterification [9]. In a round-bottom flask, a mixture of the aldehyde $(1.74 \mathrm{~g}, 5.0 \mathrm{mmol})$, 4-methoxyaniline $(0.62 \mathrm{~g}$, $5.0 \mathrm{mmol})$ and absolute ethanol $(40 \mathrm{~mL})$ was refluxed with stirring for $3 \mathrm{~h}$. The reaction mixture was filtered and the solvent was removed from the filtrate by evaporation. Recrystallization from absolute ethanol gave the title compound as a yellow solid (1.10 g, 43\%).

Melting point: $100.1{ }^{\circ} \mathrm{C}$

MS (EI): $m / z$ (rel. int. \%) = $509(1)\left(\mathrm{M}^{+}\right), 243(100)$.

IR (KBr, cm ${ }^{-1}$ ): 3449 (O-H), 2955, 2918, 2849 (C-H aliphatic); 1759 (C=O ester); 1624 (C=N); 1605, $1510(\mathrm{C}=\mathrm{C}$ aromatic $)$.

${ }^{1} \mathrm{H}$ NMR (400 MHz, $\mathrm{CDCl}_{3}$ ): $\delta / p p m ~ 0.91\left(\mathrm{t}, 3 \mathrm{H}, J=7.0 \mathrm{~Hz}, \mathrm{CH}_{3}\right), 1.27-1.44\left\{\mathrm{~m}, 28 \mathrm{H}, \mathrm{CH}_{3}\left(\mathrm{C}_{2}\right)_{14^{-}}\right\}$, 1.78 (quint, $2 \mathrm{H}, J=7.4 \mathrm{~Hz},-\mathrm{C}_{2} \mathrm{CH}_{2} \mathrm{COO}-$ ), 2.58 (t, $2 \mathrm{H}, J=7.5 \mathrm{~Hz},-\mathrm{C}_{2} \mathrm{COO}-$ ), 3.85 (s, $3 \mathrm{H}, \mathrm{OCH}_{3}$ ), $6.70(\mathrm{dd}, 1 \mathrm{H}, J=2.2,8.4 \mathrm{~Hz}, \mathrm{Ar}-\mathrm{H}), 6.76(\mathrm{~d}, 1 \mathrm{H}, J=2.2 \mathrm{~Hz}, \mathrm{Ar}-\mathrm{H}), 6.96(\mathrm{dd}, 2 \mathrm{H}, J=2.1,8.9 \mathrm{~Hz}$, Ar-H), 7.28 (dd, 2H, J=2.1, 8.9 Hz, Ar-H), 7.37 (d, 1H, J=8.3 Hz, Ar-H), 8.60 (s, 1H, CH=N), 13.76 $(\mathrm{s}, 1 \mathrm{H}, \mathrm{OH})$.

${ }^{13} \mathrm{C} \mathrm{NMR}\left(100 \mathrm{MHz}, \mathrm{CDCl}_{3}\right): \delta / p p m 172.2(\mathrm{COO}), 159.9(\mathrm{CH}=\mathrm{N}), 162.8,159.3,154.5,141.5,133.1$, 122.7, 117.7, 115.0, 113.1, 110.7 (aromatic carbons), $55.93\left(\mathrm{Ar}-\mathrm{OCH}_{3}\right), 34.85\left(-\mathrm{CH}_{2} \mathrm{COO}-\right), 25.31$ (- $\left.\underline{\mathrm{CH}}_{2} \mathrm{CH}_{2} \mathrm{COO}-\right), 32.33,30.10,30.07,30.01,29.87,29.78,29.67,29.50,23.10\left(\mathrm{CH}_{3}\left(\mathrm{CH}_{2}\right)_{14}\right)^{-}, 14.53$ $\left(\mathrm{CH}_{3}\left(\mathrm{CH}_{2}\right)_{14}\right)$.

Elemental analysis: Calculated for $\mathrm{C}_{32} \mathrm{H}_{47} \mathrm{NO}_{4} \mathrm{C}, 75.40 \%, \mathrm{H}, 9.29 \%, \mathrm{~N}, 2.75 \%$; Found: C, 75.29\%, H, $9.37 \%, \mathrm{~N}, 2.81 \%$.

\section{Acknowledgements}

The author (S.T. Ha) would like to thank Universiti Tunku Abdul Rahman (UTAR) for the UTAR Research Fund and research facilities.

\section{References and Notes}

1. Hadjoudis, E.; Vittorakis, M.; Moustakali-Mavridis, I. Photochromism and thermochromism of schiff bases in the solid state and in rigid glasses. Tetrahedron 1987, 43, 1345-1360. 
2. Hadjoudis, E.; Rontoyianni, A.; Ambroziak, K.; Dziembowska, T.; Mavridis, I.M. Photochromism and thermochromism of solid trans-N,N'-bis(salicylidene)-1,2cyclohexanediamines and trans-N,N'-bis-(2-hydroxynaphylidene)-1,2-cyclohexanediamine. J. Photochem. Photobiol. A Chem. 2004, 162, 521-530.

3. Oshima, A.; Momotake, A.; Arai, T. Photochromism, thermochromism, and solvatochromism of naphthalene-based analogues of salicylideneaniline in solution. J. Photochem. Photobiol. A Chem. 2004, 162, 473-479.

4. Yeap, G.Y.; Ha, S.T.; Ishizawa, N.; Suda, K.; Boey, P.L.; Mahmood, W.A.K. Synthesis, crystal structure and spectroscopic study of para substituted 2-hydroxy-3-methoxybenzalideneanilines. J. Mol. Struct. 2003, 658, 87-99.

5. Nair, S.M.; Bhattacharya, I. Synthesis and physiological activities of some imines and their $\beta$-lactams. Asian. J. Chem. 2009, 21, 504-510.

6. Yeap, G.Y.; Ha, S.T.; Lim, P.L.; Boey, P.L.; Ito, M.M.; Sanehisa, S.; Youhei, Y. Synthesis, physical and mesomorphic properties of Schiff's base esters containing ortho-, meta- and parasubstituents in benzylidene-4'-alkanoyloxyanilines. Liq. Cryst. 2006, 33, 205-211.

7. Ha, S.T.; Ong, L.K.; Wong, J.P.W.; Yeap, G.Y.; Lin, H.C.; Ong, S.T.; Koh, T.M. Mesogenic Schiff's base ether with dimethylamino end group. Phase Transit. 2009, 82, 387-397.

8. Ha, S.T.; Ong L.K.; Ong, S.T.; Yeap, G.Y.; Wong, J.P.W.; Koh, T.M.; Lin, H.C. Synthesis and mesomorphic properties of new Schiff base esters with different alkyl chains. Chin. Chem. Lett. 2009, 20, 767-770.

9. Ha, S.T.; Ong, S.T.; Chong, Y.T.; Yeap, G.Y. Synthesis of 4-\{[(3-chlorophenyl)imino]methyl $\}$-3hydroxyphenyl myristate. Molbank 2009, 2009, M629.

(C) 2010 by the authors; licensee MDPI, Basel, Switzerland. This article is an Open Access article distributed under the terms and conditions of the Creative Commons Attribution license (http://creativecommons.org/licenses/by/3.0/). 